\title{
GRADO Y DIRECCIÓN DE LA DIVERSIFICACIÓN DE LAS EMPRESAS INDUSTRIALES ESPAÑOLAS: UN ANÁLISIS DE LA ESTRATEGIA DE DIVERSIFICACIÓN RELACIONADA
}

\author{
DEGREE AND DIRECTION OF THE DIVERSIFICATION OF SPANISH \\ MANUFACTURING FIRMS: AN ANALYSIS OF THE STRATEGY OF RELATED \\ DIVERSIFICATION
}

\author{
Patricia Huerta Riveros ${ }^{1} \quad$ José Emilio Navas López ${ }^{2}$ \\ Recibido 22 de septiembre de 2005, aceptado el 16 de agosto de 2006 \\ Received: September 22, 2005 Accepted: August 16, 2006
}

\begin{abstract}
RESUMEN
El objetivo de este artículo es, en primer lugar, analizar brevemente las formas de medida de la diversificación corporativa a través de un análisis comparativo de éstas y, en segundo lugar, descubrir cuáles son los motivadores de la diversificación relacionada de las empresas industriales españolas. Para ello, inicialmente se realizó una revisión de la literatura especializada sobre los tipos de medida y posteriormente se estudiaron en profundidad dos teorías: La Teoría de Economía Industrial y la Teoría de Recursos y Capacidades, de las cuales existen sólidos argumentos que justifican que las empresas se diversifiquen hacia mercados relacionados. Para contrastar el modelo se utilizó la base de datos de la Encuesta sobre Estrategias Empresariales (ESEE), para el periodo comprendido entre el año 1991 y el año 2002 y se aplicó regresión logística binomial a un panel completo de observaciones de empresas diversificadas. Los resultados del estudio arrojaron que la concentración industrial, la rentabilidad industrial y los activos intangibles son factores que determinan que la empresa se diversifique hacia mercados relacionados a su negocio tradicional.
\end{abstract}

Palabras clave: Diversificación, diversificación relacionada, diversificación no relacionada, formas de medida, grado de diversificación, dirección de la diversificación.

\section{ABSTRACT}

The objective of this article is to firstly analyze the forms of measurement of corporate diversification through a comparative analysis of existing measures and secondly, to understand the motivations of Spanish manufacturing companies to perform relational diversification. To this end, a preliminary literature review on forms of measurement was conducted and then, two theories were studied: The Theory of Industrial Economy and the Theory of Resources and Capabilities. These theories provided a solid foundation for explaining why companies diversify to related markets. To test the model, data was obtained from a database that contains information about the Survey of Managerial Strategies (ESEE), for the period between 1991 and 2002. Binomial logistic regression was performed on a complete set of observations of diversified manufacturing companies. The results of the study showed that industrial concentration, industrial profitability, and intangible assets are factors that determine company diversification toward related markets.

Keywords: Diversification, related diversification, non related diversification, measurement, diversification degree, diversification direction.

\section{INTRODUCCIÓN}

La diversificación de la empresa es un tópico relevante desde el punto de vista de la Dirección de Empresas que ha sido tratado abundantemente en la literatura académica. En efecto, la problemática referida a cómo las empresas deciden acerca de los distintos negocios en los que quieren competir, es un campo de estudio de interés para la práctica empresarial que ha intentado ser resuelto desde la investigación teórica.

\footnotetext{
1 Departamento de Administración y Auditoría, Facultad de Ciencias Empresariales, Universidad del Bío-Bío, Avenida Collao 1202, Casilla 5-C, Concepción-Chile, Teléfono: 56-41-2731271, Fax: 56-41-2731087, e-mail: phuerta@ubiobio.cl

2 Departamento de Organización de Empresas, Facultad de Ciencias Económicas y Empresariales, Universidad Complutense de Madrid, Campus de Somosaguas 28223 - Pozuelo de Alarcón, Madrid-España, Teléfono: 34-913942505, Fax: 34-913942371.
} 
Como es sabido, según Boy et al. [7] una explicación común para la diversificación de las empresas es la continua búsqueda del crecimiento, pero asociado a este argumento, se encuentra la elección de la dirección de dicho crecimiento, de esta forma, cuando una empresa decide diversificarse, los nuevos negocios en los que se compromete pueden o no guardar relación con los anteriores, de forma que se suele distinguir entre diversificación relacionada y no relacionada, según se den unas u otras circunstancias, respectivamente. Sin embargo, en los últimos años se observa una cierta tendencia hacia la especialización en el diseño de las carteras de negocio, acentuada a su vez por una determinada "vuelta atrás" en el proceso de diversificación empresarial, generado por la dinámica de reestructuración de los grandes conglomerados, por lo que no cabe duda de que en la actualidad el fenómeno de la diversificación sigue siendo una práctica relevante en la realidad de las empresas y, por lo tanto, sujeto a interés académico.

Este interés se ha centrado, de forma quizás prioritaria, alrededor de dos grandes tópicos que han interesado tanto a los investigadores del área de la Dirección de Empresas como a los profesionales y ejecutivos responsables de su aplicación práctica: la forma de medir la diversificación de la empresa y la relación entre la diversificación y los resultados empresariales. Sin embargo, según Ramanujan et al. [39] mientras estos dos campos han copado gran parte de los trabajos sobre diversificación, se ha dedicado menor interés al estudio de los motivos que llevan a las empresas a seguir una estrategia de diversificación. Por esta razón, el objetivo de este artículo es, en primer lugar, realizar un breve análisis comparativo de las diferentes formas de medida de la diversificación para posteriormente determinar la forma de medida a utilizar y, en segundo lugar, descubrir cuáles son los motivadores o razones que poseen las empresas industriales españolas para seguir una estrategia de diversificación relacionada.

De la revisión de la literatura sobre las distintas perspectivas teóricas que abordan el tema de la diversificación se deduce que la Teoría de Economía Industrial y la Teoría de Recursos y Capacidades son perspectivas que permiten identificar los motivadores o factores explicativos que conducen a las empresas a seguir una estrategia de diversificación relacionada frente a una estrategia de diversificación no relacionada, por lo tanto, en base a estas dos teorías se diseña el modelo bajo estudio, el cual se contrasta utilizando como técnica estadística la regresión logística binomial para un panel completo de observaciones de empresas diversificadas en una muestra de observaciones de empresas industriales españolas, a partir de la información disponible en la base de datos de la Encuesta sobre Estrategias Empresariales (ESEE), para el periodo 1991-2002.

\section{MARCO TEÓRICO DEL ESTUDIO}

\section{Concepto y tipologías de diversificación}

A pesar de la gran cantidad de aportaciones al estudio de la diversificación persiste una cierta ambigüedad o confusión sobre el tema. De hecho, no existe una definición o medida del concepto de diversificación que sea generalmente aceptada por todos los investigadores según Rumelt [41]. En efecto, una revisión de la literatura revela que existe gran variedad en la forma como la diversificación es conceptualizada y también medida. Específicamente, para Ramanujan et al. [39] la diversificación es: "La entrada de una empresa o unidades de negocios hacia nuevas líneas de actividad, a través del desarrollo de procesos de negocios internos o adquisición, lo que ocasiona cambios en su estructura administrativa, sistemas y otros procesos directivos". En cambio, Pitts et al. [37] utilizan el término diversidad para describir "la extensión a través de la cual las empresas son simultáneamente activas en varios negocios distintos".

Por último, Suárez [46] define la diversificación empresarial como: "Las decisiones tendentes a ampliar y/o hacer más diverso el ámbito de actuación de la empresa". En concreto, estas definiciones reflejan la falta de consenso respecto a la definición del concepto de diversificación. Por lo tanto, por una parte, los investigadores señalan que no existe consenso sobre la definición de diversificación $y$, por otro lado, surgen diferentes tipologías que tratan de clasificar las distintas estrategias de diversificación seguidas por las empresas. Estas clasificaciones corresponden a Ansoff [2] y Rumelt [41].

En particular, una de las tipologías más conocidas y extendidas de las estrategias básicas de crecimiento se debe a Ansoff [3], quien fundamenta su clasificación en la relación que existe entre la situación actual o tradicional de la empresa y los nuevos desarrollos que se refieren tanto a producto como a mercado. De esta forma, Ansoff [3] identifica dos estrategias básicas: la estrategia de expansión y la estrategia de diversificación. En particular, para Ansoff [1] la estrategia de diversificación consiste en que la empresa añade simultáneamente nuevos productos y nuevos mercados a los ya existentes. Sin embargo, aunque la estrategia de diversificación supone la entrada en nuevos mercados con nuevos productos, éstos pueden o no estar relacionados de alguna forma con los actuales. Por lo tanto, a partir de esta consideración Ansoff [3] distingue cuatro 
estrategias de diversificación: Diversificación horizontal, diversificación vertical, diversificación concéntrica y diversificación conglomerada. De manera similar, bajo el enfoque de Dirección Estratégica surge otra tipología cuya clasificación original se debe a Wrigley [55], pero que posteriormente Rumelt [41] desagrega y populariza. Siendo su principal aporte la distinción entre negocio único, negocio dominante, diversificación relacionada y diversificación no relacionada. En concreto, para Rumelt [42] la diversificación es relacionada cuando existen recursos compartidos entre los negocios, canales de distribución similares, mercados comunes, tecnologías compartidas o, en definitiva, cualquier intento tangible de explotar de forma conjunta factores de producción. En cambio, la diversificación es no relacionada cuando implica un mayor grado de ruptura con la situación actual, puesto que los nuevos productos y mercados no mantienen relación alguna con los tradicionales de la empresa.

En conclusión, para efectos de este artículo una empresa diversificada será aquella que: "Participe simultáneamente en más de una industria, influyendo en el campo de actividad de la empresa y, por ende, en su estrategia corporativa o global".

\section{Análisis comparativo de las formas de medida de la diversificación}

Al comienzo, se expuso que no existe consenso sobre las definiciones de diversificación y un caso similar ocurre con las formas de medida, pese a la abundante literatura sobre el tema. En concreto, cuando se estudian las formas de medidas sobre la diversificación se suelen agrupar en dos grandes bloques: medidas continuas y medidas categóricas. Las primeras se basan en los sistemas de clasificación industrial de las actividades económicas en las cuales se encuentra la empresa. Dentro de estas medidas se puede mencionar el índice de contabilidad de productos, el índice de entropía y el espectro de diversidad amplio y estrecho. Siendo las principales ventajas: a) $\mathrm{Su}$ naturaleza objetiva y la facilidad de cálculo o medición, b) El menor tiempo y costo requeridos para la clasificación de la empresa. Sin embargo, estas medidas no están exentas de limitaciones.
Específicamente, es criticable la definición de las distintas actividades a la que da lugar, y aunque permiten distinguir entre diversificación relacionada y no relacionada, agrupa los productos por similaridades en función de la tecnología productiva o en función de cuáles sean sus principales inputs. Por lo tanto, sólo considera las relaciones existentes de tipo productivo o, en todo caso, tecnológicas, obviando cualquier otro tipo de relación. En cambio, las medidas categóricas se basan en la apreciación del analista acerca del grado de la diversificación de la empresa y el tipo de relación que existe entre los negocios de la misma.

Estas medidas fueron desarrolladas principalmente por especialistas en el área de estrategia empresarial, son de naturaleza cualitativa y permiten distinguir el tipo de estrategia de diversificación que poseen las organizaciones. Sin embargo, su principal inconveniente según Ramanujan et al. [39] es su subjetividad, ya que requiere la introducción de juicios por parte de la persona que clasifica, tanto para definir cuáles son los negocios de la empresa como el grado de relación o proximidad existente entre ellos, lo cual puede ocasionar que diferentes investigadores puedan llegar a clasificar a las empresas de distinta forma como señalan Pitts et al. [37]. La medida categórica más utilizada es la clasificación estratégica de Wrigley [55] y Rumelt [41]. La tabla 1 presenta un análisis comparativo de algunas de las principales formas de medida de la diversificación:

\section{Estudio de la diversificación relacionada a partir de la Teoría de Economía Industrial y la Teoría de Recursos y Capacidades}

Tras el análisis comparativo de las formas de medida de la diversificación se realizó una revisión de la literatura y se ha descubierto que existen dos teorías de referencia fundamental que se manifiestan como más relevantes para explicar con mayor claridad la diversificación relacionada: la Teoría de Economía Industrial y la Teoría de Recursos y Capacidades. 


\begin{tabular}{|c|c|c|c|c|}
\hline Medida & Autor & Fórmula /Descripción & Fortaleza & Debilidad \\
\hline $\begin{array}{l}\text { Contabilización de productos } \\
\text { (Índice de Berry-Herfindahl } \\
\text { modificado) }\end{array}$ & $\begin{array}{l}\text { Montgomery } \\
\text { (1982: 304) }\end{array}$ & $\begin{array}{l}\text { Indicador utilizado: } \\
\quad \text { Div. }=1-\left[\sum \mathrm{jm}_{\mathrm{j}}^{2} /\left(\sum \mathrm{jm}_{\mathrm{j}}\right)^{2}\right] \\
\text { Donde } \mathrm{m}_{\mathrm{j}}=\text { Porcentaje de ventas totales de } \\
\text { la empresa que son atribuidas al mercado } \mathrm{j} ; \\
\mathrm{j}=\text { mercado } \mathrm{j} \text {, es medido para } 2,3 \text { y } 4 \text { dígitos } \\
\text { SIC. }\end{array}$ & Facilidad de cálculo & $\begin{array}{l}\text { No mide la relación entre grupos } \\
\text { diferentes para ambos niveles de } \\
2 \text { y } 4 \text { dígitos SIC }\end{array}$ \\
\hline Índice de entropía & $\begin{array}{l}\text { Palepu } \\
(1985: 252-253)\end{array}$ & $\begin{array}{l}\text { Indicador utilizado: } \\
\qquad \begin{array}{l}\text { DT }=\mathbf{D R}+\mathbf{D N R} \\
\mathrm{DT}=\sum_{\mathrm{j}=1}^{\mathrm{M}} \mathrm{DR}_{\mathrm{j}} \mathrm{P}^{\mathrm{j}}+\sum_{\mathrm{j}=1}^{\mathrm{M}} \mathrm{P}^{\mathrm{j}} \ln \left(1 / \mathrm{P}^{\mathrm{j}}\right) \\
\text { Donde } ; \mathrm{M}=\text { Número de grupos de industria; } \mathrm{j}=1 . . . \\
\mathrm{M} ; \mathrm{P}=\text { Proporción de las ventas del grupo } \mathrm{j} \text {-ésimo, } \\
\text { en el total de ventas de la empresa. }\end{array}\end{array}$ & $\begin{array}{l}\text { Captura la diversidad a través de } \\
\text { grupos de productos (relacionados) } \\
\text { y dentro de grupos de productos (no } \\
\text { relacionados). Calcula la cantidad } \\
\text { de diversificación total y sus } \\
\text { componentes. } \\
\text { - Diversificación Relacionada } \\
\text { (DR), } \\
-\quad \text { Diversificación No Relacionada } \\
\text { (DNR) }\end{array}$ & $\begin{array}{l}\text { Requiere información de ventas } \\
\text { a nivel de } 4 \text { dígitos } \\
\text { Complejidad de cálculo }\end{array}$ \\
\hline $\begin{array}{l}\text { Espectro de diversidad amplio } \\
\text { (extenso) o estrecho (reducido) }\end{array}$ & $\begin{array}{l}\text { Varadarajan y } \\
\text { Ramanujan } \\
(1987: 382)\end{array}$ & $\begin{array}{l}\text { Indicadores utilizados: } \\
\text { BSD: número de códigos de dos dígitos SIC en } \\
\text { el cual una empresa participa. } \\
\text { MNSD: número de códigos de cuatro dígitos } \\
\text { SIC en el que una empresa participa dividido } \\
\text { por el número de categorías de dos dígitos SIC } \\
\text { en que la empresa opera. }\end{array}$ & $\begin{array}{l}\text { Simplicidad } \\
\text { Facilidad de cálculo }\end{array}$ & $\begin{array}{l}\text { Validez y fiabilidad } \\
\text { cuestionable }\end{array}$ \\
\hline Clasificación de Wrigley/Rumelt & $\begin{array}{l}\text { Wrigley (1970); } \\
\text { Rumelt (1974) }\end{array}$ & $\begin{array}{l}\text { Indicadores utilizados: } \\
\text { Ratio de especialización (RE); Ratio de Relación } \\
\text { (RR); Ratio Vertical (RV). } \\
\text { Categorías definidas: Negocio Único; Negocio } \\
\text { Vertical Dominante; Negocio Dominante; Negocio } \\
\text { Relacionado; Negocio No Relacionado. }\end{array}$ & $\begin{array}{l}\text { Rigor conceptual } \\
\text { Considera la historia de la empresa } \\
\text { al igual que su comportamiento } \\
\text { para determinar la utilización de sus } \\
\text { fortalezas, habilidades esenciales y } \\
\text { sus objetivos de diversificación }\end{array}$ & $\begin{array}{l}\text { Subjetividad } \\
\text { Fiabilidad cuestionable } \\
\text { Necesidad de tiempo para el } \\
\text { análisis } \\
\text { Necesidad de información } \\
\text { abundante } \\
\text { Necesidad de varias fuentes de } \\
\text { información }\end{array}$ \\
\hline
\end{tabular}

3 Para un análisis más detallado de las formas de medida de la diversificación ver Huerta et al. [17]. 
En general, estas dos teorías ${ }^{4}$ se pueden considerar complementarias, ya que ambas explican las fuentes de ventajas competitivas de las empresas. De hecho, según Penrose [34], la primera plantea que tales fuentes se encuentran en la estructura de la industria, y la segunda señala que son los recursos y capacidades que poseen las empresas los que explican los rendimientos superiores. De manera similar, se considera que los móviles del crecimiento pueden estar fuera de ella (en el ámbito externo) o bien dentro de la empresa (en su ámbito interno). De esta forma, el estímulo a la diversificación puede aparecer del entorno, en forma de una oportunidad de negocio generadora de rentas que la empresa es capaz de identificar. O bien el incentivo a la diversificación puede ser inicialmente interno y surgir de la existencia de unos recursos inactivos para los que se busca su aprovechamiento. En este segundo caso, determinados recursos de la empresa, pese a no encontrarse realmente inactivos, son transferidos hacia otro uso en el que son más productivos. En definitiva, la diversificación se verá propiciada por la existencia de unos activos actualmente infrautilizados, pero valiosos en alguna actividad distinta a la original. Por lo tanto, ambas teorías hacen referencia a los recursos que son compartibles entre los negocios, por ello esta es una de las razones por las cuales se estudian ambas teorías para descubrir los motivadores de la diversificación hacia mercados relacionados.

En particular, la Economía Industrial es un área de investigación económica que se ha ocupado ampliamente del fenómeno de la diversificación empresarial. Según Segarra [44] es una disciplina del análisis económico que estudia la naturaleza de las industrias y las características de los mercados donde operan las empresas y, más concretamente, de las causas y las consecuencias que se derivan de la presencia de poder de mercado. Se asocia al estudio de determinadas características del sector, como el grado de concentración, ritmo de crecimiento, barreras de entrada, etc. Por ende, al estudiar la diversificación, según Suárez [46] ésta se considera no como un tipo de estrategia sino como uno de los atributos de un sector. Bajo este planteamiento el argumento base será que: "participar en más de un negocio permite a las empresas aumentar su poder de mercado en cada uno de ellos".

\footnotetext{
4 Existen otras teorías, tales como la Teoría Neoclásica, la Teoría de Costes de Transacción, la Teoría de la Agencia y la Teoría de Gestión del Conocimiento, las cuales también han estudiado el proceso de diversificación empresarial, pero no serán utilizadas en esta investigación porque consideramos que la Teoría de Economía Industrial y la Teoría de Recursos y Capacidades poseen argumentos más sólidos para justificar la estrategia de diversificación relacionada a través de sus motivadores o factores explicativos.
}

En concreto, el poder de mercado es la habilidad de un participante o grupo de participantes para influenciar el precio, la calidad y la naturaleza de los productos en el mercado. Específicamente, el planteamiento de poder de mercado es posible para una empresa diversificada indiferente de su dirección, relacionada o no relacionada, de manera que las empresas diversificadas pueden aumentar o extender su poder de mercado a través de diferentes mecanismos que no se encuentran a disposición de las empresas especializadas como: Subsidios cruzados, precios predatorios, reciprocidad en compradores y proveedores y barreras de entrada.

Sin embargo, cuando la empresa opera en varios mercados a través de diferentes unidades estratégicas de negocio, esto le permitirá influir en el potencial de rivalidad existente en la industria y por ende en su poder de mercado. De hecho, una empresa diversificada puede responder a un ataque en su industria atacando las otras industrias en las que participa obteniendo, de esta forma, una reputación de represalias en el sector. Por lo tanto, la competencia multiindustria reduce el potencial de rivalidad en el sector. A la vez, se debe considerar que en la medida que el tipo de diversificación sea más evidente, es decir, hacia industrias relacionadas, la posibilidad de colusión será mayor, y por lo tanto, reducirá la rivalidad en las industrias en las cuales opera la empresa permitiéndole, por ende, aumentar su poder de mercado en cada uno de los negocios que posee.

De esta forma, Montgomery [24] argumentó que las empresas con diversificación no relacionada tienen bajo poder de mercado en sus respectivas industrias en relación con las empresas con diversificación relacionada. Por ello, estas últimas presentan mayor poder de mercado en sus respectivas industrias y, por lo tanto, mayores cuotas de mercado y mayor concentración, en relación con las empresas con diversificación no relacionada. A la vez, su estudio demostró que las empresas con diversificación no relacionada no tienen fuertes posiciones de mercado, al igual que, en promedio, ellas compiten en industrias menos atractivas en relación con las empresas que poseen diversificación relacionada. En base en estos argumentos se plantea la hipótesis $\mathrm{N}^{\mathrm{o}} 1$.

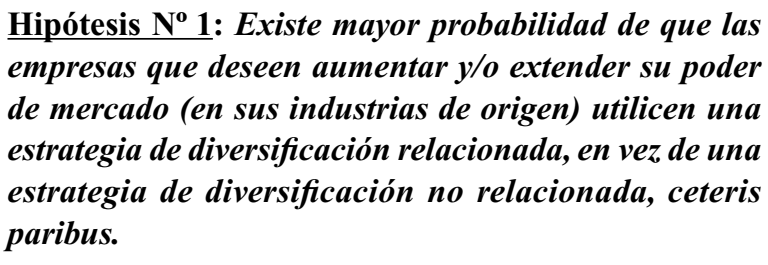


De acuerdo con Montgomery [24], los factores que contribuyen a aumentar el poder de mercado son la cuota de mercado y la concentración industrial, por lo que se analizará individualmente cada uno de ellos. La cuota de mercado es el porcentaje de participación de la empresa en su industria base, tal como lo señala Lecraw [19], o el promedio ponderado de la cuota de mercado de la empresa en sus mercados como afirman Merino et al. [22]. Desde el punto de vista de la diversificación, Christensen et al. [10] demostraron que las empresas con diversificación relacionada limitada tienen índices de cuota de mercado ponderadas mayores y las empresas con diversificación no relacionada poseen índices de cuotas de mercado ponderadas menores. De manera similar, Montgomery [24] argumentó que las empresas con diversificación relacionada presentan mayor poder de mercado en sus respectivos mercados y específicamente que estas empresas poseen mayor cuota de mercado en relación con las empresas con diversificación no relacionada. Por esta razón, se plantea la hipótesis $1 \mathrm{~A}$.

\section{Hipótesis No 1A: Existe mayor probabilidad de que las empresas que posean mayor cuota de mercado (en sus industrias de origen) utilicen una estrategia de diversificación relacionada, en vez de una estrategia de diversificación no relacionada, ceteris paribus.}

Para Clarke [11], una industria se encuentra más concentrada cuanto menor es el número de empresas en la producción o cuanto más desigual es la distribución de las cuotas de mercado, considerando constantes otros factores. Por lo tanto, la concentración industrial hace referencia al grado en el cual la producción se encuentra concentrada en manos de un reducido número de grandes empresas en una industria determinada. Según Cuervo [12] este proceso de concentración puede deberse a múltiples factores, entre los cuales cabe citar el que una empresa pueda crecer más que los competidores como reflejo de una mayor competitividad o mayor capacidad innovadora, por la regulación de la industria, donde el gobierno establece un número limitado de licencias o permisos para operar que limitan la entrada de nuevos competidores, y a través de fusiones y adquisiciones buscando, más que supuestas economías de escala y alcance, facilitar la reestructuración empresarial y reducir el exceso de capacidad productiva de muchos sectores.

Específicamente, Christensen et al. [10] encontraron que las empresas con diversificación relacionada limitada se ubicaban en mercados más concentrados. Similar es la evidencia empírica obtenida por Montgomery [24] quien argumentó que las empresas con diversificación relacionada presentan mayor poder de mercado en sus respectivos mercados y, específicamente, que los mercados de estas empresas presentan mayor concentración industrial en relación con las empresas con diversificación no relacionada. Por ello, se plantea la hipótesis $1 \mathrm{~B}$.

Hipótesis No 1B: Existe mayor probabilidad de que las empresas situadas en industrias más concentradas (en sus industrias de origen) utilicen una estrategia de diversificación relacionada, en vez de una estrategia de diversificación no relacionada, ceteris paribus.

A la vez, Christensen et al. [10] y también Bettis [5] confirmaron que la diversificación relacionada fue asociada con una alta rentabilidad en relación con la diversificación no relacionada y que la categoría diversificada relacionada limitada posee un mejor desempeño en relación con las otras categorías. Sin embargo, estos autores atribuían las diferencias en el desempeño a las características de las industrias en las que se ubicaban las empresas de mejor desempeño. Específicamente, Christensen et al. [10] observaron que las empresas de la categoría relacionada limitada operaban en mercados más rentables, mientras que Bettis [5] atribuyó su rentabilidad superior a la presencia de cuatro compañías farmacéuticas entre ellas, según Grant et al. [16]. Por otra parte, cuando la rentabilidad industrial aumenta, los motivos de los directivos para utilizar una diversificación defensiva ${ }^{5}$ disminuyen, mientras que los motivos para utilizar la diversificación ofensiva ${ }^{6}$ aumentan, según Park [31,32]. La diversificación defensiva está siendo asociada a la diversificación no relacionada y la diversificación ofensiva a la diversificación relacionada, ambas basadas en la rentabilidad de la industria en la cual se encuentra presente la empresa. Por lo tanto, la alta rentabilidad industrial favorece la diversificación ofensiva $y$, por ende, aumenta la tendencia de la empresa a seguir una estrategia de diversificación relacionada.

\footnotetext{
5 Según Weston et al. [54] cuando una industria tiene baja rentabilidad y además no es atractiva estructuralmente, es más probable que los directivos de las empresas sigan una "diversificación defensiva". La diversificación defensiva, intenta evitar un desarrollo desfavorable en las industrias tradicionales de la empresa. Rumelt [41] también sugirió que la diversificación es un medio para escapar de las expectativas de declinación de los negocios originales. Por lo tanto, los directivos de una empresa en una industria que no es atractiva estructuralmente o que posea baja rentabilidad industrial tienden a tener fuertes motivos para mejorar la expectativa global de la empresa diversificándose hacia industrias más atractivas. Sin embargo, cuando una industria no es rentable o atractiva, las industrias en las cuales las empresas podrían entrar a través de la diversificación relacionada, es también probable que no sean rentables o atractivas, según Christensen et al. [10]. Por ende, como señala Park [31,32] las empresas en una industria de baja rentabilidad tienden a diversificarse hacia industrias no relacionadas.

6 Para Yip [56] la diversificación ofensiva se refiere a la diversificación que explota recursos y capacidades desarrollados en las industrias actuales de la empresa.
} 
Park [31, 32] afirma que la rentabilidad industrial promedio reflejará el atractivo estructural de la industria, donde la industria o estructura de mercado es a menudo definida como poseedora de ciertos atributos estables del mercado que definen el marco competitivo entre los participantes. Por lo tanto, los altos niveles de rentabilidad industrial indicarán la presencia de barreras estructurales en la industria, según los argumentos de Wernerfelt et al. [53]. Así, las empresas que se encuentren en una industria con alta rentabilidad industrial tenderán a seguir diversificación relacionada en lugar de una estrategia de diversificación no relacionada.

Específicamente, Christensen et al. [10] demostraron que las empresas con diversificación no relacionada se ubicaban en mercados menos rentables y, al contrario, los diversificadores relacionados limitados se ubicaban en mercados más rentables, en relación con las otras empresas de la muestra. De manera similar, Montgomery [24] argumentó que los mercados de las empresas con diversificación relacionada presentaban mayores niveles promedios de rentabilidad industrial en relación con los mercados de las empresas con diversificación no relacionada. Por estas razones, se plantea la hipótesis $\mathrm{N}^{\circ} 2$. La tabla 2 recoge los motivadores o razones que poseen las empresas para seguir diversificación relacionada, desde la Teoría de Economía Industrial.

Hipótesis $N^{\circ}$ 2: Existe mayor probabilidad de que las empresas situadas en industrias (de origen) con alta rentabilidad industrial utilicen una estrategia de diversificación relacionada, en vez de una estrategia de diversificación no relacionada, ceteris paribus.

Al contrario, desde la perspectiva de la Teoría de Recursos y Capacidades, el crecimiento de la empresa se explica por la existencia de activos no utilizados o infrautilizados en un determinado momento. Esta menor utilización puede deberse a que los recursos distintivos de la empresa son en muchos casos indivisibles o, al menos, no perfectamente divisibles, por lo que la distinta velocidad en su uso provoca capacidades sobrantes. Por otra parte, los recursos sobrantes pueden tener múltiples usos, es decir, pueden ser empleados en los negocios actuales, o bien en nuevos negocios, lo que justificaría los procesos diversificadores. Sin embargo, para Teece [50] la posesión de capacidad sobrante de algún recurso productivo que no pueda ser empleada en los negocios tradicionales no es condición suficiente para que se produzca la diversificación empresarial, ya que la empresa puede venderla en el mercado.

En definitiva, y siguiendo este argumento según Suárez [46]: "La empresa se diversificará si existe algún tipo de imperfección en el mercado que hace que el intercambio conlleve unos costos de transacción muy altos, mayores que los costos de explotar ese recurso internamente", o bien, según Montgomery [25] y Dewan et al. [13]: "las empresas se diversificarán en respuesta al exceso en factores productivos o de recursos que no pueden ser transferidos a través de los límites de la empresa sin incurrir en altos coste de transacción", es decir, como argumentan Montgomery et al. [27], que se encuentren sujetos a imperfecciones del mercado.

Por lo tanto, bajo el planteamiento de la Teoría de Recursos y Capacidades la justificación a la diversificación de las empresas se encuentra en compartir recursos y capacidades estratégicos entre los negocios, tal como afirman Penrose [33], Mahoney et al. [21], Peteraf [36], Robins et al. [40] y Teece et al. [51], o bien, en dar uso a los recursos ociosos, en aquellos casos en que los mercados no funcionan satisfactoriamente, según Teece [49]. Además, desde la teoría de los recursos se postula que la "diversificación hacia negocios relacionados" es la que tiene mayor racionalidad, puesto que transfiriendo factores valiosos, se conseguirá replicar las capacidades originales.

Tabla 2. Razones para seguir diversificación relacionada (Fuente: Elaboración propia).

\begin{tabular}{|c|c|}
\hline Razones & Autor \\
\hline \multicolumn{2}{|r|}{ Economía Industrial } \\
\hline \multirow{3}{*}{$\begin{array}{l}\text { 1. Poder de mercado } \\
\text { 1.1. Cuota de mercado } \\
\text { 1.2. Concentración Industrial }\end{array}$} & $\begin{array}{l}\text { Montgomery (1985: 789); Palepu (1985: 241); Suárez (1993: 141); Palich, } \\
\text { Cardinal y Miller (2000: 156-157); Segarra (2001: 25) }\end{array}$ \\
\hline & Christensen y Montgomery (1981: 331); Montgomery (1985: 791) \\
\hline & $\begin{array}{l}\text { Christensen y Montgomery (1981: 331); Montgomery (1985: 791); } \\
\text { Clarke (1993: 23) }\end{array}$ \\
\hline 2. Rentabilidad Industrial & $\begin{array}{l}\text { Christensen y Montgomery (1981: 331); Porter (1982: 23-24); Montgomery } \\
\text { (1985: 791); Park (2002: 1004-1005; 2003: 473) }\end{array}$ \\
\hline
\end{tabular}


Por ende, para Loredo [20] se debe considerar que la relación entre los distintos sectores no es en sí misma una garantía para el éxito de la estrategia de diversificación, porque son los recursos los que en última instancia determinarán los resultados empresariales. Por lo tanto, que la diversificación se oriente hacia un negocio relacionado, únicamente facilita que se puedan transvasar los "recursos" y reproducir las "capacidades" iniciales. En consecuencia, si una empresa no posee recursos compartidos entre sus negocios que puedan considerarse "específicos", se esperará que valga menos que la suma de sus negocios por separado, tal como lo afirma Forcadell [14].

De esta forma, la Teoría de los Recursos y Capacidades enlazando el pensamiento estratégico tradicional con las contribuciones del nuevo análisis económico aporta una explicación adicional en la que los recursos y capacidades de la empresa constituyen la unidad básica de análisis. Así, para Chatterjee et al. [9] desde esta perspectiva integradora, la oportunidad para diversificarse surge cuando la empresa posee recursos sobrantes o infrautilizados, cuyo valor es más alto en otros negocios distintos de aquél en donde se originaron, tal como lo afirman Suárez, [47] y Loredo [20], que tienen múltiples usos y para los cuales existe una imperfección en el mercado, según los argumentos de Peteraf [36], Suárez [47] y Dewan et al. [13]. Por lo tanto, como señala Montgomery et al. [27] las fallas del mercado hacen de la diversificación una elección eficiente. De acuerdo a los argumentos anteriores, se plantea la hipótesis $\mathrm{N}^{\mathrm{o}} 3$ :

\section{Hipótesis $\mathrm{N}^{\circ}$ 3: Existe mayor probabilidad de que las empresas que presenten un exceso de recursos utilicen una estrategia de diversificación relacionada, en vez. de una estrategia de diversificación no relacionada, ceteris paribus.}

En concreto, según Suárez et al. [48] las características y configuraciones de los recursos disponibles son predictores más adecuados para justificar la entrada en nuevos negocios frente a otros factores del entorno. De hecho, Silverman [45] señala que la base de los recursos de una empresa afecta la elección de las industrias hacia las cuales las empresas diversifican, y Montgomery et al. [26] plantean que las empresas tienden a entrar en mercados donde los requerimientos de los recursos son similares a las características de los recursos que poseen las empresas. Por su parte, Montgomery et al. [27] señalan que la estrategia de diversificación que siga una empresa depende de los recursos específicos que posea.

En general, desde el punto de vista de la diversificación, Montgomery et al. [27] señalan que son los recursos menos específicos los que pierden menos eficiencia a medida que son empleados en negocios cada vez más alejados de los originales. Además, estos factores normalmente obtienen una menor ventaja competitiva porque poseen una mayor oferta. Por lo tanto, los recursos menos específicos normalmente apoyan una diversificación amplia (es decir, una diversificación no relacionada) y, por ende, su valor (relativo) más bajo tiende a fortalecer la relación negativa entre la extensión de la diversificación y la renta promedio. Al contrario, se supone que las empresas que poseen recursos más específicos se diversificarán con mayor probabilidad en negocios próximos o similares a los originales a través de una "estrategia de diversificación relacionada". Por lo tanto, dada la especificidad de un conjunto de factores, la decisión óptima para una empresa es aplicar su exceso de capacidad hacia las oportunidades de entrada más cercanas.

En general, la literatura ha categorizado usualmente los recursos que afectan a las decisiones de diversificación, en tres clases de recursos según Chatterjee et al. [9] y Chatterjee et al. [8]: a) Recursos físicos, b) Recursos intangibles, y c) Recursos financieros. En concreto, Chatterjee et al. [9] argumentan que, por una parte, los dos primeros tipos de recursos (físicos e intangibles) son bastante más inflexibles y, por lo tanto, pueden ser utilizados para entrar sólo en "mercados estrechamente relacionados". Y de manera similar, Chatterjee et al. [8] y Teece [50] señalan que los recursos físicos tales como las plantas, los equipos, etc., y los recursos intangibles, como el conocimiento específico en investigación y desarrollo o de mercado, son recursos bastante específicos o inflexibles, por lo tanto, sólo pueden ser utilizados para expandirse hacia "mercados relacionados".

Respecto a los recursos financieros, según Chatterjee et al. [8] éstos no son específicos, o bien, son recursos que poseen más flexibilidad y, por lo tanto, se encontrarán a disposición de cualquier estrategia, o más concretamente, por ser menos específico será más cercano a la estrategia de diversificación no relacionada. Por ello, Chatterjee et al. [9] plantean que los recursos físicos e intangibles llevarán a una diversificación más relacionada, mientras que los recursos financieros llevarán a cualquier tipo de diversificación. A la vista de la mayor especificidad de los recursos físicos e intangibles se plantean las hipótesis 3 A y 3 B. La tabla 3 presenta los motivadores o razones que poseen las empresas para seguir una estrategia de diversificación relacionada.

Hipótesis No 3A: Existe mayor probabilidad de que las empresas que presenten un exceso de recursos físicos utilicen una estrategia de diversificación relacionada, en 
vez de una estrategia de diversificación no relacionada, ceteris paribus.

Hipótesis No 3B: Existe mayor probabilidad de que las empresas que presenten un exceso de recursos intangibles utilicen una estrategia de diversificación relacionada, en vez de una estrategia de diversificación no relacionada, ceteris paribus.

\section{FUENTES DE INFORMACIÓN Y MEDIDAS DE LAS VARIABLES}

La base de datos utilizada en este estudio es la Encuesta Sobre Estrategias Empresariales (ESEE), que depende de la Fundación Sepi en España. El periodo elegido de análisis es de 1991 a $2002 .{ }^{7}$ Para este periodo de 12 años se determinó el número de observaciones totales de empresas que responden a la encuesta a través de un panel completo de empresas. Se trabajó con un conjunto de panel completo para lograr una mayor homogeneidad en la muestra, identificando sólo aquellas empresas que siempre responden a la encuesta en el periodo bajo análisis.

Para ello se creó una variable llamada "panel", la cual es una variable dicotómica que toma el valor " 1 " en aquellos casos en los cuales las empresas responden a la ESEE en los 12 años del estudio y "0" en caso contrario. Con ello se pretende reunir un conjunto de "observaciones de empresas diversificadas comunes" para el horizonte temporal considerado.
En concreto, para la determinación de la muestra se partió con 10.716 observaciones de empresas, de las cuales se descontaron los valores perdidos que fueron 922 observaciones de empresas y, a la vez, también se restaron aquellas observaciones de empresas que siguen una estrategia de especialización. Como resultado se obtuvo una muestra de 1.554 observaciones de empresas diversificadas desde 1991 hasta el año 2002. Con un error muestral del 0,02 a un nivel de confianza del 95\%.

Para la contrastación del modelo se utilizó el programa estadístico SPSS 12, y la técnica estadística utilizada fue la regresión logística binomial, puesto que nuestra variable dependiente es dicotómica.

Es necesario considerar que la base de datos utilizada (ESEE) no entrega información de los sectores industriales, más allá de dos dígitos $\mathrm{CNAE}$, hacia los cuales las empresas diversifican, por considerar dicha información como secreto estadístico, por ello se ha elaborado en colaboración con la Fundación Sepi un índice que mide tanto el grado como la dirección de la diversificación de las empresas a partir de la base de datos utilizada. Este índice identifica tres categorías: Especialización, diversificación relacionada y diversificación no relacionada. Específicamente, este índice permite discriminar entre empresas especializadas y diversificadas, y dentro de estas últimas, entre empresas con diversificación relacionada y no relacionada. En esta investigación se utilizaron para contrastar las hipótesis estas dos últimas categorías. Esta medida es una transformación de datos ya validados por la ESEE, lo cual garantiza la validez del índice de diversificación utilizado.

Tabla 3. Razones para seguir diversificación relacionada (Fuente: Elaboración propia).

\begin{tabular}{|l|l|}
\hline \multicolumn{2}{|c|}{ Teoría de Recursos y Capacidades } \\
\hline \multicolumn{1}{|c|}{ Razones } & \multicolumn{1}{c|}{ Autores } \\
\hline $\begin{array}{l}\text { 1.- Exceso de recursos } \\
\text { 1.1.- Exceso de recursos físicos } \\
\text { 1.2.- Exceso de recursos intangibles }\end{array}$ & $\begin{array}{l}\text { Montgomery y Wernerfelt (1988: 623); Chatterjee y Wernerfelt (1991: } \\
\text { 33); Peteraf (1993: 188); Montgomery (1994: 167); Suárez (1994: 106); } \\
\end{array}$ \\
& Dewan, Michael y Min (1998: 221) \\
& Chatterjee y Wernerfelt (1991: 35); Suárez (1993: 146); Chatterjee y \\
& Singh (1999: 30) \\
\hline & Chatterjee y Wernerfelt (1991: 35); Suárez (1993: 146); Dewan, Michael \\
& y Min (1998: 219-222); Chatterjeee y Singh (1999: 30); Silverman (1999: \\
& $1123) ;$ Forcadell (2000: 2-6); Park (2002: 1005) \\
\hline
\end{tabular}

7 En el momento de realización del trabajo sólo se encontraban disponibles los datos hasta el año 2002. 
El índice mencionado anteriormente se ha creado analizando los códigos de clasificación industrial en el cual participan las empresas a nivel de tres dígitos y a partir de ellos se asignó la empresa a una categoría estratégica de la siguiente forma: Si la empresa sólo define un producto a nivel de tres dígitos de códigos CNAE, se señala que la empresa no diversifica, es decir, es una empresa especializada y en este caso se le asignó el número "0", en cambio, si la empresa define un producto a nivel de tres dígitos, y esta empresa se encuentra en el mismo sector a dos dígitos, como por ejemplo: 263, 264, 265, la empresa tendrá una diversificación relacionada y, por lo tanto, se le asignó el número "1", por último, la empresa que define más de un producto a nivel de tres dígitos, y algunos de éstos se encuentran en distinto sector a dos dígitos, como por ejemplo 263, 264, 325, la empresa tendrá una diversificación no relacionada y se le asignó el número "2".

Considerando las categorías anteriores se construyó la variable dependiente, la cual sólo reúne las observaciones de empresas diversificadas, de tal forma que la empresa tomará el valor "1" cuando posee una "estrategia de diversificación relacionada", y tomará el valor " 0 " en caso contrario, es decir, cuando posea una "estrategia de diversificación no relacionada". ${ }^{8}$

Desde la Teoría de Economía industrial se han identificado como variables independientes el poder de mercado, el cual se define a través de la concentración industrial y la cuota de mercado, y también la rentabilidad industrial. Específicamente, se entenderá como concentración industrial al grado de concentración en el mercado principal, medida por la suma de las cuotas de mercado de las cuatro primeras empresas de ese mercado. Sin embargo, como esta variable se construye a partir de la percepción de la empresa sobre cuáles son las principales cuatro empresas competidoras en su mercado, se calcula un promedio de tales valores. En cambio, la cuota de mercado cual se mide por el porcentaje que representa la cuota de mercado aproximada de la empresa en el mercado principal de venta de sus productos. Por último, la rentabilidad industrial será el promedio del margen bruto de explotación de las empresas en la industria.

En cambio, desde la Teoría de Recursos y Capacidades se identificó como variables independientes, el exceso de recursos físicos e intangibles. El exceso ${ }^{9}$ de recursos

8 Se ha realizado una recodificación de la diversificación no relacionada pasando del número "2" al número "0" para crear parte de la variable dependiente.

9 Las variables de exceso (tangible e intangible) se construyen como una diferencia entre la variable de la empresa y el promedio de las empresas en la industria, con el objetivo de reflejar el exceso. Específicamente, se tomó la variable de la empresa y se comparó físicos: se midió a través de la capacidad ociosa, estimada por la diferencia del porcentaje de utilización de la capacidad instalada de la empresa. Y el exceso de recursos intangibles: se calcula por el exceso de gastos en investigación y desarrollo, definidos por el gasto de I + D de la empresa sobre ventas.

Por otra parte, las variables control más utilizadas en los estudios sobre diversificación son el tamaño y el sector industrial. Sin embargo, no se ha introducido como variable control la variable sector, por encontrarse recogida en la forma en la que se han construido las variables a través de diferencias con las medias sectoriales. Por ello, sólo se ha considerado como variable control a la variable tamaño, medida por el número promedio anual de trabajadores de la empresa. Esta variable se ha dicotomizado para ser coherentes con la forma en la cual la base de datos utilizada recoge la información, la cual distingue entre empresas de más de doscientos trabajadores y empresas de menor o igual a doscientos trabajadores.

\section{MODELO EMPÍRICO}

La tabla 4 refleja el modelo econométrico del estudio. En este modelo se intenta determinar la probabilidad que una empresa siga una estrategia de diversificación relacionada. En efecto, la probabilidad que la empresa "i" que está en el mercado “j” se diversifique a " $k$ " dependerá de la cuota de mercado de la empresa "i" en el mercado "j", de la concentración industrial en el mercado "j”, de la rentabilidad en el mercado " $\mathrm{j}$ ”, del exceso de recursos físicos de la empresa "i" en el mercado " $j$ ", del exceso de recursos intangibles de la empresa " $i$ " en el mercado “j”, y del mayor tamaño de la empresa "i" en el mercado “j”, más un error $\varepsilon$.

\section{RESULTADOS}

En el análisis se han llevado a cabo tres regresiones. En la regresión 1, se consideran sólo las variables industriales (Concentración Industrial, Rentabilidad Industrial) y la

\footnotetext{
en relación con el promedio de las empresas en la industria y posteriormente se volvió a comparar la variable de la empresa, pero en esta ocasión en relación con el promedio de las empresas diversificadas en la industria. Si la diferencia es positiva, es decir, si la variable de la empresa supera la media, se supondrá que presenta exceso, en caso contrario, es decir, si no supera la media, no presentará exceso. Inclusive, en ambos casos se consideró para su construcción el año y el sector principal al que pertenecía cada observación. Además, se ha optado por construir las variables a través de diferencias, para restar el efecto del sector, por lo que no se han introducido "dummies sectoriales" en el estudio.
} 
variable de control (Tamaño). En la regresión 2 se incluyen las variables Cuota de Mercado, Exceso de Recursos Tangibles (capacidad ociosa) y Exceso de Recursos Intangibles $(\mathrm{I}+\mathrm{D})$ en relación con el promedio de la industria. En la regresión 3 se consideran las variables industriales y de control y, a la vez, también se incluyen las variables Cuota de Mercado, Exceso de Recursos Tangibles (capacidad ociosa) y Exceso de Recursos Intangibles (I+D), pero en esta ocasión, en relación al promedio de las empresas diversificadas en la industria, buscando con ello un mayor ajuste en el análisis.

En concreto, a la vista de los resultados de la tabla 5, en la regresión 1 observamos el comportamiento de las variables industriales y la variable control utilizada en nuestro estudio. El objetivo de esta regresión es mostrar el resultado que arrojan las variables que son comunes en las regresiones posteriores (regresiones 2 y 3 ) pero de una manera aislada. De la tabla, se puede deducir que existe una relación positiva y significativa en las variables concentración industrial y rentabilidad industrial con la decisión de seguir una estrategia de diversificación relacionada, resultado consistente con los planteamientos derivados del modelo propuesto. Además, se aprecia que no existe un resultado significativo con la variable tamaño.

En la regresión 2 se han introducido todas las variables del estudio, de las cuales tres de ellas se encuentran construidas en relación con el promedio de la industria (cuota de mercado y exceso de recursos tangibles e intangibles). Específicamente, se aprecia que existe nuevamente una relación positiva y fuertemente significativa en las variables, concentración industrial, rentabilidad industrial y la variable investigación y desarrollo en relación con el promedio de las empresas en la industria. Sin embargo, la variable cuota de mercado fue significativa pero negativa con la decisión de seguir una estrategia de diversificación relacionada.

Tabla 4. Especificación del modelo (Elaboración propia).

$$
\begin{aligned}
& \operatorname{Pr}\left(D_{\mathrm{ijk}}=1\right)=\beta_{0}+\beta_{1} \text { Cuota de } \text { Mercado }_{\mathrm{ij}}+\boldsymbol{\beta}_{2} \text { Concentración Ind. } \mathrm{j}+\boldsymbol{\beta}_{3} \text { Rentabilidad Ind.j }+\boldsymbol{\beta}_{4} \text { Exceso Recursos }
\end{aligned}
$$

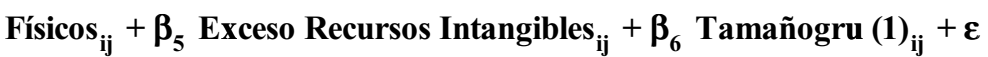

Tabla 5. Resultados de la estimación del modelo de regresión logística binomial para observaciones de panel completo (Elaboración propia).

\begin{tabular}{|l|c|c|c|}
\hline \multirow{2}{*}{ Variables } & Regresión 1 & Regresión 2 & Regresión 3 \\
\cline { 2 - 4 } & Coef. (Wald) & Coef. (Wald) & Coef. (Wald) \\
\hline Cuota de Mercado & - & $-0,005^{* *}(4,205)$ & $-0,007 * * * *(7,562)$ \\
\hline Concentración Industrial & $0,018^{* * * *}(14,386)$ & $0,018^{* * * *}(11,864)$ & $0,017 * * * *(11,075)$ \\
\hline Rentabilidad Industrial & $0,059^{* * * *}(20,557)$ & $0,048^{* * * *}(12,918)$ & $0,049^{*} * * *(13,689)$ \\
\hline Capacidad Ociosa & - & $0,000(0,000)$ & $0,003(0,619)$ \\
\hline $\mathrm{I}+\mathrm{D}$ & - & $5,358^{* *}(3,882)$ & $2,013(1,057)$ \\
\hline Tamaño (1) & $0,007(0,004)$ & $0,012(0,011)$ & $0,050(0,180)$ \\
\hline Constante & $-1,545^{* * * *}(41,761)$ & $-1,427 * * * *(31,592)$ & $-1,436 * * *(32,250)$ \\
\hline-2 log. Verosimilitud & 2075,902 & 1821,359 & 1821,384 \\
\hline Chi-cuadrado & $36,034 * * *$ & $35,162 * * * *$ & $35,136^{* * * *}$ \\
\hline $\mathrm{R}^{2}$ Cox y Snell & 0,023 & 0,025 & 0,025 \\
\hline $\mathrm{R}^{2}$ Nagelkerke & 0,031 & 0,034 & 0,034 \\
\hline Pr. Hosmer y Lemeshow & 3,849 & 10,115 & 5,895 \\
\hline $\mathrm{N}$ & 1.554 & 1.367 & 1.367 \\
\hline$\%$ clasificación correcto & $55,4 \%$ & $55,3 \%$ & $55,8 \%$ \\
\hline DR vs. DNR & $55,5 \%$ vs. $55,4 \%$ & $54,7 \%$ vs. $55,8 \%$ & $55,4 \%$ vs. $56,1 \%$ \\
\hline
\end{tabular}

$* * * * \mathrm{p}<0,001 ; * * * \mathrm{p}<0,01 ; * * \mathrm{p}<0,05 ; * \mathrm{p}<0,1$. 
De manera similar, en la regresión 3 se realiza el análisis considerando estas tres últimas variables pero en relación con las empresas diversificadas. Nuevamente las variables concentración industrial, rentabilidad industrial vuelven a aparecer como positivas y fuertemente significativas. Sin embargo, al contrario del resultado de la regresión anterior, la variable investigación y desarrollo deja de ser significativa. Resumiendo, se puede concluir según los resultados que los motivadores o razones de las empresas para seguir una estrategia de diversificación relacionada para un conjunto de observaciones de panel completo de empresas industriales españolas son: La concentración industrial y la rentabilidad industrial. De esta forma, se puede afirmar que existirá mayor probabilidad de que las empresas que se ubiquen en industrias más concentradas y rentables sigan una estrategia de diversificación relacionada.

Además, como se mencionó, un factor adicional que presenta la muestra es la investigación y desarrollo en relación con el promedio de las empresas en la industria. De esta forma, se puede afirmar que existirá mayor probabilidad de que las empresas que posean un exceso de investigación y desarrollo en relación con la industria tenderán a seguir una estrategia de diversificación relacionada. Por otra parte, la tabla 3 también recoge cinco formas de evaluar la bondad del ajuste del modelo: Prueba Ómnibus sobre los coeficientes del modelo (Chi-cuadrado), -2 logaritmo de la verosimilitud, $\mathrm{R}^{2}$ de Cox y Snell, $\mathrm{R}^{2}$ de Nagelkerke y la Prueba de Hosmer y Lemeshow. En cuanto a la Prueba Ómnibus, esta medida ofrece una prueba de ajuste global del modelo, a través del estadístico Chi-cuadrado $\left(\chi^{2}=36,034 ; \chi^{2}=35,162\right.$ y $\chi^{2}=35,136$ para las regresiones 1,2 y 3 , respectivamente), como se observa la mejora es fuertemente significativa $(p<0.001)$ en las tres regresiones del modelo propuesto. Por lo tanto, se puede rechazar la hipótesis nula y concluir que la incorporación de las variables mejora significativamente el ajuste y capacidad predictiva del modelo.

Por otra parte, el resto de medidas, tales como -2 logaritmo de la verosimilitud, $\mathrm{R}^{2}$ de Cox y Snell y el $\mathrm{R}^{2}$ de Nagelkerke muestran los estadísticos de bondad de ajuste para el modelo estimado. Sin embargo, puesto que el modelo de regresión logística utiliza como variable dependiente una variable categórica, estos estadísticos son meramente orientativos, incluso suelen adoptar valores moderados o incluso bajos aun cuando el modelo estimado pueda ser apropiado y útil, según Pardo et al. [30].

Con estos tres estadísticos se puede valorar el ajuste global de modelo en el paso 1, es decir, el modelo que incluye todas las variables. El valor de -2 logaritmo de la verosimilitud es de 2075,902; 1821,359 y 1821,384, respectivamente, indicando un mejor ajuste de la regresión 2 frente al resto. Sin embargo, si consideramos el resto de medidas, tales como el $\mathrm{R}^{2}$ de Cox y Snell y el $\mathrm{R}^{2}$ de Nalgerkerke se puede deducir que los resultados son similares en las tres regresiones, y además son considerablemente bajos. Lo anterior se debe a que son resultados en general para las empresas manufactureras españolas y no son análisis más detallado por sectores industriales, lo cual contribuiría a mejorar los $\mathrm{R}^{2}$, tal como lo hemos comprobado al realizar regresiones por sectores. Sin embargo, ya se ha mencionado que estos resultados son meramente orientativos. Paralelamente, la Prueba de Hosmer y Lemeshow arroja un valor no significativo al $5 \%$ en todas las regresiones, por 10 tanto, se puede rechazar la hipótesis nula de igualdad de distribuciones y, en consecuencia, se puede asumir que la regresión 2 se ajusta mejor a los datos.

Por último, también se ha introducido el número de observaciones con las cuales se ha calculado la regresión en cada caso y el porcentaje de clasificación correcta. En general, se puede observar que se está trabajando con aproximadamente todas las observaciones de la muestra y el porcentaje de clasificación de observaciones correctas es superior al $50 \%$ en todos los casos.

\section{CONCLUSIONES Y DISCUSIÓN FINAL}

La diversificación de la empresa es uno de los temas relevantes de estudio de diferentes áreas de conocimiento (finanzas, marketing, dirección estratégica, economía industrial), los cuales en su mayoría se han concentrado en investigar la relación que existe entre la diversificación y los resultados y las formas de medida de la diversificación, presentando menor atención a los motivos que tienen las empresas para seguir un proceso de diversificación.

En esta investigación se ha realizado, en primer lugar, un análisis comparativo de las diferentes formas de medida de la diversificación y, posteriormente, se ha llevado a cabo un análisis de la literatura académica para descubrir cuáles son los motivadores o razones que poseen las empresas para seguir una estrategia de diversificación relacionada, a partir del estudio de dos teorías que poseen mayor poder explicativo: La Teoría de Economía Industrial y Teoría de Recursos y Capacidades.

En primer lugar, el análisis comparativo ha servido para descubrir que no existe consenso sobre la forma de medida (al igual que la definición de la diversificación), pero su 
principal contribución fue conocer el estado actual de las formas de medida para posteriormente construir el índice de medida utilizado. Esta medida actualmente es de uso público y se encuentra disponible a través de la base de datos empleada en esta investigación.

En segundo lugar, en base a la Teoría de Economía Industrial y la Teoría de Recursos y Capacidades se ha construido el modelo de análisis de los motivadores de la diversificación relacionada. Dicho modelo se ha contrastado con la información proporcionada por la Encuesta sobre Estrategias Empresariales, para un periodo de doce años, utilizando como método estadístico la regresión logística binomial. Para llevar a cabo las regresiones se construyó un panel completo de datos, con el objetivo de observar el comportamiento de las mismas empresas a lo largo del tiempo. Específicamente, se ha encontrado apoyo empírico para la hipótesis $1 \mathrm{~B}$, la hipótesis 2 y la hipótesis 3B. Por lo tanto, se pueden confirmar estas hipótesis y señalar que existirá mayor probabilidad de que las empresas que se encuentren en industrias más concentradas y rentables sigan una estrategia de diversificación relacionada.

De manera similar, se puede señalar que existirá mayor probabilidad de que las empresas que posean exceso de recursos intangibles (o investigación y desarrollo) seguirán una estrategia de diversificación relacionada. De esta forma, se supone que las empresas que presenten exceso de recursos intangibles tenderán a utilizar o trasladar dichos recursos en o hacia sus negocios relacionados. A la vez, se ha rechazado la hipótesis $1 \mathrm{~A}$, por lo tanto, se puede señalar que existirá menor probabilidad de que las empresas que posean una cuota de mercado mayor sigan una estrategia de diversificación relacionada, resultado contrario a lo que se esperaba según la argumentación teórica. Por último, en este estudio la variable tamaño no fue significativa, pese a ser considerada una variable relevante en los estudios previos de diversificación; para este extraño resultado existe una explicación que se recoge a partir del análisis de la base de datos utilizada, la cual discrimina entre empresas grandes y pequeñas. En concreto, cuando esta base de datos recoge información se observa que la mayoría de las empresas que se mantienen en el tiempo son las empresas de mayor tamaño, por lo tanto, el tamaño en esta muestra de panel completo de observaciones de empresas diversificadas no fue una variable que discrimine, esta es una línea de investigación que se pretende seguir estudiando posteriormente.
Por otra parte, una limitación de esta investigación es la utilización de información de panel completo de observaciones de empresas, porque con esta técnica lamentablemente se pierde en eficiencia econométrica al descartar aquellas observaciones de empresas que no se mantienen los 12 años del estudio, pero al contrario, se gana en la observación del comportamiento de las mismas empresas a lo largo del tiempo. Otra limitación se asocia a la forma de medida utilizada porque aunque permite distinguir entre diversificación relacionada, no relacionada y empresas especializadas, esta clasificación se realiza a partir de códigos de clasificación industrial, los cuales no reconocen otro tipo de relación que no sea productivo o tecnológico para asignar a una empresa a una determinada categoría estratégica, lo cual no permite descubrir si existe otra fuente de relación en la cual se pueda explotar sinergia, siendo la sinergia la justificación tradicional de las empresas para seguir una estrategia de diversificación relacionada.

\section{AGRADECIMIENTOS}

El autor agradece a la Universidad del Bío-Bío, Concepción, Chile, por colaborar en la realización de esta investigación, a través de la aprobación de un proyecto de investigación interno. Al Dr. José Emilio Navas López, profesor del Departamento de Organización de Empresas de la Universidad Complutense de Madrid, España. A la Fundación SEPI quienes han colaborado a través de la Encuesta sobre Estrategias Empresariales, Madrid, España.

\section{REFERENCIAS}

[1] H. Ansoff. "Strategies For Diversification", Harvard Business Review. Vol. 35 N 5, pp. 113124. 1957.

[2] H. Ansoff. "Corporate Strategy". Penguin. Harmonds-Worth. 1965.

[3] H. Ansoff. "La Estrategia de la Empresa". Universidad de Navarra. Pamplona, España. 1976.

[4] C. Berry. "Corporate Growth And Diversification". Princeton University Press. Princeton. 1975.

[5] R. Bettis. "Performance Differences In Related and Unrelated Diversified Firms". Strategic Management Journal. Vol. 2, pp. 379-393. 1981. 
[6] Booz, Allen and Hamilton. "Diversification: A Survey of European Chief Executives". Booz, Allen and Hamilton. Inc. Nueva York. 1985.

[7] B. Boy, S. Gove and M. Hitt. "Consequences of Measurement Problems in Strategic Management Research: The Case of Amihud and Lev". Strategy Management Journal. Vol. 26, pp. 367-375. 2005.

[8] S. Chatterjee and J. Sing. "Are Tradeoffs Inherent In Diversification Moves? A Simultaneous Model for Type of Diversification and Mode of Expansion Decisions". Management Science. Vol. 45 No 1 , pp. 25-41. 1999.

[9] S. Chatterjee and B. Wernerfelt. "The Link Between Resources and Type of Diversification: Theory and Evidence". Strategic Management Journal. Vol. 12, pp. 33-48. 1991.

[10] H. Christensen and C. Montgomery. "Corporate Economic Performance: Diversification Strategy Versus Market Structure". Strategic Management Journal. Vol. 2, pp. 327-343. 1981.

[11] R. Clarke. "Economía Industrial”. Colegio de Economistas de Madrid. Madrid, España. 1993.

[12] A. Cuervo. "Dinámica Empresarial y Consolidación Sectorial”. Universia Business Review”. No 1, pp. 96-105. 2004.

[13] S. Dewan, S. Michael and C. Min. "Firm Characteristics and Investments in Information Technology: Scale and Scope Effects". Information Systems Research. Vol. 9 N 3, pp. 219-232. 1998.

[14] F.J. Forcadell. "Diversificación e Intangibles Tecnológicos a partir del Enfoque Basado en los Recursos: Una Aplicación a las Empresas Industriales Españolas”. X Congreso Nacional ACEDE. Oviedo, España. 2000.

[15] M. Gort. "Diversification and Integration in American Industry". Princeton University Press. Princeton. 1962.

[16] R. Grant and A. Jammine. "Performance Differences Between the Wrigley/Rumelt Strategic Categories". Strategic Management Journal. Vol. 9, pp. 333346. 1988.
[17] P. Huerta, J. Navas y P. Martínez. "Cómo Medir la Diversificación Corporativa: Una Aplicación a las Empresas Industriales Españolas". Teoría. Vol. 13, pp. 59-68. 2004.

[18] M. Kamien and N. Schwartz. "Market Structure and Innovation: A Survey". Journal of Economic Literature. Vol. 13, pp. 1-37. 1975.

[19] D. Lecraw. "Diversification Strategy and Performance”. Journal of Industrial Economics. Vol. 3, pp. 179-198. 1984.

[20] E. Loredo. "Diversificación Relacionada y Transferencia de Recursos: Un Análisis de Caso". IX Congreso Nacional ACEDE. Burgos, España. 1999.

[21] J.T. Mahoney and J.R. Pandian. "The ResourceBased view within the Conversation of Strategic Management". Strategic Management Journal. Vol. 13, pp. 363-380. 1992.

[22] F. Merino De Lucas and D. Rodríguez. "A Consistent Analysis of Diversification Decisions With NonObservable Firm Effects". Strategic Management Journal. Vol. 18 No 9, pp. 733-743. 1997.

[23] C. Montgomery. "The Measure of Firm Diversification: Some New Empirical Evidence", Academy of Management Journal. Vol. $25 \mathrm{~N}^{\circ} 2$, pp. 299-307. 1982.

[24] C. Montgomery. "Product - Market Diversification and Market Power". Academy of Management Journal. Vol. 8, pp. 789-798. 1985.

[25] C. Montgomery. "Corporate Diversification". Journal of Economic Perspectives. Vol. $8 \mathrm{~N}^{\circ} 3$, pp. 163-178. 1994.

[26] C. Montgomery and S. Hariharan. "Diversified Expansion By Large Established Firms”. Journal of Economic Behavior and Organization. Vol. 15, pp. 71-89. 1991.

[27] C. Montgomery, B. Wernerfelt. "Diversification, Ricardian, Rents, and Tobin's Q". Rand Journal of Economics. Vol. 19, pp. 623-632. 1988.

[28] K. Palepu. "Diversification Strategy. Profit Performance and the Entropy Measure". Strategic Management Journal. Vol. 6, pp. 239-255. 1985. 
[29] L. Palich, L. Cardinal and C. Miller. "Curvilinearity in the Diversification-Performance Linkage: An Examination of Over Three Decades of Research". Strategic Management Journal. Vol. 21, pp. 155174. 2000.

[30] A. Pardo y M. Ruiz. "Spss 11: Guía para el Análisis de Datos”. McGraw-Hill. Madrid, España. 2002.

[31] C. Park. "The Effects of Prior Performance on the Choice Between Related and Unrelated Acquisitions: Implications for the Performance Consequences of Diversification Strategy". Journal of Management Studies. Vol. $39 \mathrm{~N}^{\mathrm{o}} 7$, pp. 1003-1019. 2002.

[32] C. Park. "Research Notes and Commentaries: Prior Peformance Characteristics of Related and Unrelated Acquirers". Strategic Management Journal. Vol. 24, pp. 471-480. 2003.

[33] E. Penrose. "The Theory of the Growth of the Firm". Wiley. Nueva York, Estados Unidos. 1959.

[34] E. Penrose. "Teoría del Crecimiento de la Empresa". Aguilar. Madrid, España. 1962.

[35] M. Pérez. "La Interrelación de las Unidades Estratégicas de Negocio como Instrumento Moderador de la Eficacia en las Organizaciones. Una Aplicación Empírica en el Sector de la Construcción en España”. Tesis para optar al grado de doctor. Universidad Complutense de Madrid. Madrid, España. 1996.

[36] M. Peteraf. "The Cornerstones of Competitive Advantage: A Resource-Based View”. Strategic Management Journal. Vol. 14, pp. 179-191. 1993.

[37] R. Pitts and H. Hopkins. "Firm Diversity: Conceptualization and Measurement". Academy of Management Review. Vol. 7 No 4, pp. 620-629, 1982.

[38] M. Porter. "Estrategia Competitiva". C.E.C.S.A. México. 1982.

[39] V. Ramanujan and P. Varadarajan. "Research on Corporate Diversification: A Synthesis", Strategic Management Journal. Vol. 10, pp. 523-551. 1989.
[40] J. Robins and M. Wiersema. "A Resource-Based Approach to the Multibusiness Firm: Empirical Analysis of Porfolio Interrelationships and Corporate Financial Performance". Strategic Management Journal. Vol. 16, pp. 277-299, 1995.

[41] R. Rumelt. "Strategy, Structure, and Economic Performance". Harvard Business School Press. Boston, Estados Unidos. 1974.

[42] R. Rumelt. "Diversification Strategy and Profitability". Strategic Management Journal. Vol. 3, pp. 359-369. 1982.

[43] R. Sambharya. "Assessing the Construct Validity of Strategic and Sic-Based Measures of Corporate Diversification". British Journal of Management. Vol. 11, pp. 163-173. 2000.

[44] A. Segarra. "Mercados y Empresa" y "Barreras a la Entrada e Innovación Tecnológica". En Callejón, M. (Coordinadora). Economía Industrial. Civitas. Madrid, España. Cap. 1 y 5. 2001.

[45] B. Silverman. "Technological Resources and the Direction of Corporate Diversification: Toward an Integration of the Resources-Based View and Transaction Cost Economics". Management Science. Vol. 45 N$^{\circ}$ 8, pp. 1109-1124. 1999.

[46] I. Suárez. "Fundamentos Teóricos y Empíricos de la Relación entre Diversificación y Resultados Empresariales: Un Panorama”. Revista de Economía Aplicada. Vol. 1 No 3, pp. 139-165. 1993.

[47] I. Suárez. "Estrategia de Diversificación y Resultados de la Empresa Española". Revista de Economía Aplicada. Vol. 11 No 4, pp. 103-128. 1994.

[48] I. Suárez and J. Vicente. "Decisiones de Diversificación y Reestructuración: Reflexiones desde el Enfoque de Recursos". Cuadernos Aragoneses de Economía. Vol. 11 No 1, pp. 67-89. 2001.

[49] D. Teece. "Economies of Scope and the Scope of the Enterprise". Journal of Economic Behavior and Organization. Vol. 1, pp. 223-247. 1980.

[50] D. Teece. "Towards and Economic Theory of the Multi-Product Firm". Journal of Economic Behavior and Organization. Vol. 3, pp. 39-63. 1982. 
[51] D. Teece, G. Pisano and A. Shuen. "Dynamic Capabilities and Strategic Management". Strategic Management Journal. Vol. 18, pp. 509-533. 1997.

[52] P. Varadarajan and V. Ramanujam. "Diversification and Performance: A Reexamination Using a New Two-Dimensional Conceptualization of Diversifity in Firm". Academy of Management Journal. Vol. $30 \mathrm{~N}^{\mathrm{o}}$ 2, pp. 380-393. 1987.

[53] B. Wernerfelt and C. Montgomery. "What Is an Attractive Industry?". Management Science. Vol. 32, pp. 1223-1229. 1986.
[54] J. Weston and S. Mansinghka. "Tests of the Efficiency Performance of Conglomerate Firms". Journal of Finance. Vol. 26, pp. 916-936. 1971.

[55] L. Wrigley. "Divisional Autonomy and Diversification". Tesis para optar al grado de doctor. Harvard Business School. 1970.

[56] G. Yip. "Diversification Entry: Internal Development Versus Acquisition". Strategic Management Journal. Vol. 3, pp. 331-345. 1982. 